\title{
Umweltforschung wieder aktuell
}

$\mathrm{D}$ as menschliche Genom ist entschlüsselt, ebenso das von einer Vielzahl von Tieren und Pflanzen. Ausgelöst durch die Faszination der molekularen Genetik - und in ihr gefangen - wurde die Umweltforschung für viele junge Wissenschaftler und auch Gutachter in Forschungsförderungsgremien immer weniger attraktiv. Auf der Jagd nach der Aufschlüsselung der genetischen Grundlagen der Allergie wurde jedoch bald klar, dass es nicht ein „Atopie-Gen" gibt, sondern dass es wahrscheinlich etwa 150 verschiedene Genloci sein werden, die mit bestimmten Phänotypen in der komplexen Kaskade der allergischen Reaktion assoziiert sind und deren klinische Bedeutung erst herausgearbeitet werden muss. Nach wie vor unklar ist vor allen Dingen, warum bei gleichem Genotyp nur bestimmte Individuen tatsächlich erkranken und andere nicht. Auch die weltweit beobachtete Zunahme der Allergieprävalenz lässt sich durch die genetische Disposition alleine nicht erklären. Da kommt erneut die Umwelt ins Spiel.

Die großen Forschritte in der Molekulargenetik haben es erst möglich gemacht, die Bedingungen der Sensibilisierung, das heißt, die molekularen Grundlagen der Allergieinduzierenden Aminosäure-Sequenzen auf den Allergenen (B- und T-Zell-Epitope) $\mathrm{zu}$ erkennen (siehe M. Raulf-Heimsoth et al. und W.-M. Becker). Natürlich birgt jeder Fortschritt auch Risiken in sich, wie am Beispiel gentechnisch veränderter Lebensmittel im Hinblick auf eventuelle Nahrungsmittel-Allergien gezeigt wird (siehe W.-M. Becker). Allerdings demonstriert gerade auch dieses Beispiel, wie wichtig die Präsenz allergologischer Expertise in den entsprechenden Gremien ist, um echte Gefahren für den Menschen aufzuzeigen und erfolgreich verhindern zu können.

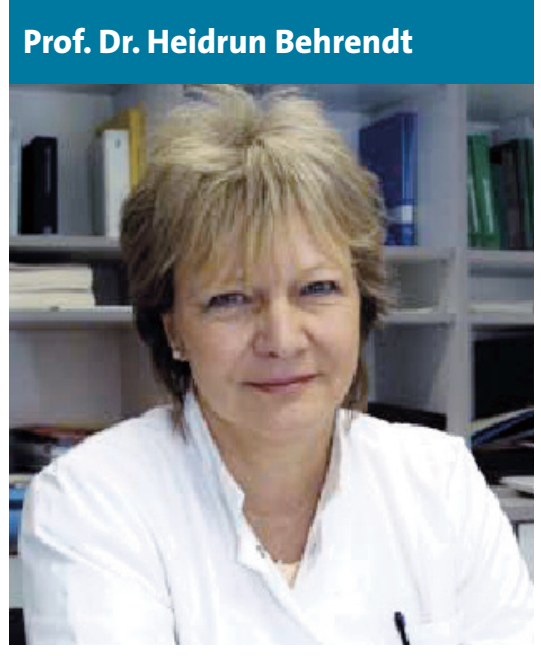

ZAUM - Zentrum Allergie und Umwelt, Technische Universität München

Allergene wirken in allen Ebenen der Allergieentwicklung, von der Sensibilisierung bis hin zur Symptomausprägung und Verstärkung, als kausales Agens. Es gibt jedoch Umwelteinflüsse die entweder „adjuvant“ oder „protektiv" modulierend die Allergieentstehung beeinflussen. Hier sind besonders die luftgetragenen anthropogenen und biogenen Substanzen zu nennen. Denjenigen, welche eine Th1-dominierte Immunantwort induzieren, z. B. Endotoxine, und protektiv sind, werden anthropogene Umweltstoffe mit Allergie-fördernder Wirkung gegenübergestellt. Während über Jahrzehnte den gasförmigen Luftschadstoffen die größte Aufmerksamkeit geschenkt wurde (Schwefeldioxid etc.), war spätestens nach der deutschen Wiedervereinigung klar, dass diese Art der Luftverschmutzung nicht mit Allergien assoziiert ist. Die aufregenden Befunde der niedrigeren Heuschnupfen- und Asthmaprävalenzen bei ostdeutschen im Vergleich zu westdeutschen Kindern haben dies klar gezeigt, leider aber auch zu dem voreiligen Schluss geführt, dass Luftschadstoffe überhaupt keine Rolle in der Allergieentstehung oder in der Zunahme der Allergieprävalenz spielen würden. Heute weiß man, dass nicht die gasförmigen, sondern vielmehr die partikulären Luftschadstoffe die entscheidende Rolle spielen: nicht die großen Staubpartikel aus dem Smog der 50er Jahre oder den Braunkohlekraftwerken Osteuropas, sondern die feinen und ultrafeinen Partikel unter 2,5 bzw. 1,0 $\mu \mathrm{m}$ (PM2,5 bzw. PM1,0) sind von ausschlaggebender Bedeutung. Am Beispiel der Dieselrußpartikel (siehe C. Lemmen et al.) konnte klar gezeigt werden, dass diese Partikel in der frühen Phase der Allergieentstehung bei gleichzeitiger Allergenbelastung eine Verschiebung zur Th2-Polarisierung hin bewirken, dies sowohl im Tierexperiment als auch in der menschlichen Zellkultur.

Es konnte gezeigt werden, dass Pollen nicht nur Allergenträger sind, sondern im feuchten Milieu auch in der Lage sind, selbst aktive Mediatoren mit proinflammatorischer, Eikosanoid-ähnlicher Wirkung („pollen associated mediators", PALMs) freizusetzen, die auf der Haut und Schleimhaut zur Attraktion und Aktivierung von Entzündungszellen beitragen. Diese Befunde könnten unser Weltbild im Hinblick auf die Frage nach der allergenen Potenz bestimmter Pflanzenspezies ebenso ändern wie im Hinblick auf mögliche Maßnahmen der Prävention.

Erst wenn wir die Mechanismen besser verstehen, über die die Umweltfaktoren (die wir ändern können) mit der genetischen Substanz eines Individuums interagieren, werden auch rationale und sinnvolle primäre Präventionskonzepte möglich sein. Genetik ist nach wie vor „in“, aber ohne moderne Umweltforschung wird es in der Allergologie nicht weitergehen!
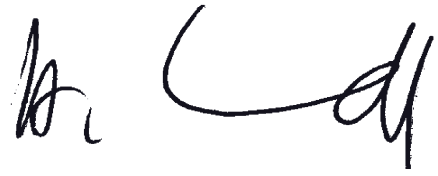

Prof. Dr. Heidrun Behrendt 